\title{
The pollen-specific DEFH125 promoter from Antirrhinum is bound in vivo by the MADS-box proteins DEFICIENS and GLOBOSA
}

Received: 23 September 2005/ Accepted: 21 November 2005/Published online: 22 December 2005

(C) Springer-Verlag 2005

\begin{abstract}
The Antirrhinum DEFH125 MADS-box protein is expressed in maturing pollen and thus likely participates in the regulation of pollen development. Here, we describe the characterization of a $2.5 \mathrm{kbp}$ promoter fragment conferring pollen-specific GUS expression in Antirrhinum, as well as in the distantly related species Arabidopsis. Taking advantage of the higher sensitivity of the diphtheria toxin A-chain $(D T A)$ reporter gene assay, onset of DEFH125 promoter activity could be defined to start at the late unicellular microspore stage. Stamen development in Antirrhinum is governed by the class B MADS-box genes DEFICIENS $(D E F)$ and $G L O B O S A(G L O)$. The respective proteins form a heterodimer and are expressed throughout stamens, except for microspores. Complementary expression patterns of $D E F H 125$ and $D E F / G L O$ during later stamen development tempted us to investigate whether the DEF/GLO heterodimer might bind the DEFH125 promoter and could thus be involved in repressing the DEFH125 expression. The ChIP technique was applied to investigate protein/DNA interactions occurring in vivo. We report the identification of a 200 bp DEFH125 promoter fragment that is in vivo bound by DEF and GLO proteins. This fragment contains a CArG-box motif, known to mediate DNA binding of MADS-box proteins. Implications for a likely function of $D E F$ and $G L O$ in the transcriptional control of DEFH125 are discussed.
\end{abstract}

Andrea Lauri and Shuping Xing contributed equally to the work.

A. Lauri $\cdot$ S. Xing $\cdot$ I. Heidmann $\cdot$ H. Saedler $\cdot$ S. Zachgo $(\bowtie)$ Max Planck Institute for Plant Breeding Research, 50829 Köln, Germany

E-mail: szachgo@mpiz-koeln.mpg.de

Present address: I. Heidmann

Enza Zaden, Research \& Department B.V.,

Enkhuizen, The Netherlands
Keywords Antirrhinum Chromatin immunoprecipitation - MADS-box protein · Pollen-specific promoter $\cdot$ Protein/DNA interaction

Abbreviations ChIP: Chromatin immunoprecipitation DEF: DEFICIENS - DEFH125: DEFICIENS HOMOLOG 125 - DTA: Diphtheria toxin A-chain . GLO: GLOBOSA - GUS: $\beta$-Glucuronidase

\section{Introduction}

Stamens are the male reproductive organs of flowering plants. In Antirrhinum, Arabidopsis and other model species, stamen organogenesis is controlled by the overlapping activity of homeotic class B and C MADSbox transcription factors (Schwarz-Sommer et al. 1990; Coen and Meyerowitz 1991). The Antirrhinum class B genes DEFICIENS (DEF) and GLOBOSA (GLO) regulate stamen and petal organogenesis (Schwarz-Sommer et al. 1992; Tröbner et al. 1992). Lack of either one of the class B function genes in Antirrhinum causes a conversion of stamens into carpels and petals into sepals (Sommer et al. 1990; Tröbner et al. 1992). Analysis of the DEF and GLO protein expression pattern showed that the expression is restricted to the second and third whorl organs and starts at early floral stages (Zachgo et al. 2000). Analysis of the subcellular protein localization detected a strong expression in the nuclei, being in accordance with their function as transcriptional activators and repressors throughout petal and stamen development (Zachgo et al. 1995; Bey et al. 2004). DEF and GLO proteins were shown to form a heterodimer that can bind to short regulatory DNA fragments, so-called conserved CArG-box motifs (Tröbner et al. 1992). After initiation of petal and stamen primordia, DEF and GLO expression is maintained by an autoregulatory loop, likely by directly binding CArG-box motifs in their own promoters as shown by in vitro binding studies (Zachgo et al. 1995). Recently, it has been shown for Antirrhinum and 
Arabidopsis that additional MADS-box proteins can interact with the class B heterodimer (Egea-Cortines et al. 1999; Honma and Goto 2001). The combinatorial activity of class $\mathrm{B} / \mathrm{C}$ genes in the control of stamen identity is thus likely exerted by the formation of multimeric MADS-box complexes (Honma and Goto 2001; Theißen and Saedler 2001).

However, little is known about the processes controlling the formation of various tissues and cell types within the stamens during further development. Differentiation of stamen primordia starts with the formation of a stalked basis, the filament, and convex protrusions on the top that give rise to the pollen sacs. Within the anther, diploid male sporogenous cells differentiate into pollen mother cells. In Arabidopsis, this process is under the control of the putative transcription factor NOZZLE/SPOROCYTELESS, as lack of function mutants fail to form pollen mother cells (Schiefthaler et al. 1999; Yang et al. 1999). The pollen mother cells undergo meiosis leading to the formation of a tetrad of cells; held together by a thick callose wall. The tapetum, a nutritive sporophytic cell layer lining the locules containing the developing microspores, releases an enzyme mixture called callase that separates the haploid microspores (Scott et al. 2004). During further microgametogenesis, microspores enlarge and undergo an asymmetric division, generating two cells with very distinct fates (McCormick 2004). The large vegetative cell accumulates dense cytoplasm containing lipids, proteins and carbohydrates to provide storage compounds for the period of pollen tube growth. The small generative cell contains highly condensed chromatin and undergoes another mitosis producing the two sperm cells.

Other transcription factors known to regulate gametophytic development like the MALE STERILE and ABORTED MICROSPORE1 gene seem to affect gametophytic development rather indirectly by regulating tapetal gene expression in Arabidopsis (Wilson et al. 2001; Sorensen et al. 2003). Overall, recent Arabidopsis microarray analysis revealed an under-representation of transcription factors during pollen development (Honys and Twell 2003, 2004; Pina et al. 2005).

Besides the well-characterized pollen-specific promoters from the tomato genes LAT52 and LAT59 (Bate and Twell 1998), there is still the need for other characterized pollen-specific promoters that confer different expression strengths or different expression windows (McCormick 2004). The protein expression of the Antirrhinum MADS-box transcription factor DEFH125 is restricted to pollen in unfertilized plants. Upon fertilization, DEFH125 protein was also detected in the transmitting tract (Zachgo et al. 1997). Here, we report on the characterization of the pollen-specific DEFH125 promoter in Antirrhinum and Arabidopsis using reporter genes of different sensitivities. Furthermore, chromatin immunoprecipitation (ChIP) experiments demonstrate an in vivo interaction of the DEF/GLO proteins at the DEFH125 promoter.

\section{Materials and methods}

\section{DEFH125 promoter analysis}

A genomic Antirrhinum phage library was screened with a DEFH125 cDNA probe as described by Zachgo et al. (1997). A $7 \mathrm{kbp}$ phage insert was isolated and sequenced containing $2.5 \mathrm{kbp}$ upstream of the putative DEFH125 start codon. For GUS expression analysis, the promoter fragment was cloned into the pGPTV-hpt vector (Becker et al. 1992). For the DEFH125::DTA cell ablation construct, the GUS cDNA was removed from the DEFH 125::GUS vector and replaced by DTA that was produced by PRC using DTA primers introducing additional restriction sites (5' CCACGAGCTCGCCTT CACAAAGATCGCCTG, SacI; 3' CAGCTCT AGAGC CATGGATCCTGATGATG, XbaI) for suitable cloning into the binary vector. DTA gene was obtained from I. Maxwell, University of Colorado Health Sciences Center, Denver, CO, USA. Constructs were transformed into Antirrhinum (ecotype 165E, MPIZ, Cologne) and Arabidopsis (Columbia, Nottingham Stock Center), following protocols from Heidmann et al. (1998) and Clough and Bent (1998), respectively. After hygromycin selection, transgenic T1 plants were analyzed.

\section{Expression studies}

GUS staining, protein immunolocalization and DAPI staining were conducted as described by Heidmann et al. (1998) and Zachgo et al. (1995). For detailed GUS analysis, transgenic plants were fixed in FAA

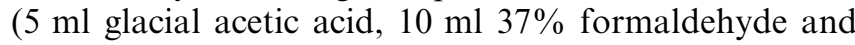
$85 \mathrm{ml} \mathrm{70 \%}$ ethanol), embedded in paraffin and sectioned. Sections from the anthers harboring the DEFH125::DTA construct were stained with $0.1 \%$ Naphthol Blue Black (Aldrich Chemical Company, Inc.). Images were captured using the digital camera KY-F70B (JVC). For pollen tube GUS staining, mature pollen were dropped on the surface of germination medium $\left(1 \mathrm{mM} \mathrm{CaCl}_{2}, 1 \mathrm{mM} \quad \mathrm{MgSO}_{4} / \mathrm{H}_{2} \mathrm{O}, 1 \mathrm{mM}\right.$ $\mathrm{KNO}_{3}, 0.01 \% \mathrm{H}_{3} \mathrm{BO}_{3}$ and $10 \%$ sucrose) and incubated for $6 \mathrm{~h}$ before adding the staining solution. Arabidopsis pollen germination was conducted as described by Chen and McCormick (1996).

\section{Chromatin immunoprecipitation experiments}

Plant fixation and processing were largely performed as described by Müller (2003). The material was fixed with $1 \%$ paraformaldehyde in $0.1 \mathrm{M} \mathrm{Na}$-phosphate buffer, $\mathrm{pH} 7.4$, for $20 \mathrm{~min}$ under vacuum and for $100 \mathrm{~min}$ on a rotating wheel at $4^{\circ} \mathrm{C}$. Fixation was stopped with $0.125 \mathrm{M}$ glycine. Samples were washed three times 10 min with washing buffer $(0.1 \mathrm{M}$ Na-phosphate buffer, $\mathrm{pH}$ 7.4, $0.125 \mathrm{M}$ glycine). 
Fixed samples were ground in liquid nitrogen. To the sample powder, $30 \mathrm{ml}$ of buffer A $(10 \mathrm{mM}$ Hepes $/ \mathrm{KOH}$, $\mathrm{pH} 7.9,10 \mathrm{mM} \mathrm{KCl}, 1.5 \mathrm{mM} \mathrm{MgCl}_{2}, 0.5 \mathrm{mM}$ DTT, $0.1 \%$ NP-40, $10 \%$ glycerol) including $200 \mu \mathrm{l}$ proteinase inhibitor (Sigma P-9599) was added. The slurry was filtered successively through $300,75,20$ and $10 \mu \mathrm{m}$ nylon membranes and nuclei were collected by $10 \mathrm{~min}$ centrifugation at $500 \mathrm{~g}$. The nuclei pellet was washed five times with $10 \mathrm{ml}$ buffer $\mathrm{A}$, then transferred into a $2 \mathrm{ml}$ eppendorf tube. Nuclei were resuspended in $450 \mu$ sonication buffer $(10 \mathrm{mM}$ Hepes/KOH, pH 7.9, $100 \mathrm{mM}$ $\mathrm{NaCl}, 1 \mathrm{mM}$ EDTA, pH 8.0, 1 mM EGTA, pH 8.0, $0.1 \%$ Triton $\mathrm{X}-100, \quad 0.1 \%$ sodium deoxycholate, $0.125 \mathrm{M}$ glycine). Chromatin was solubilized by sonication using a Hielscher UP50H sonicator (Dr. Hielscher GmbH, Teltow, Germany) with an amplitude setting of $60 \%$ and a cycle control of 0.65 for 20 times, each $10 \mathrm{~s}$. Immunoprecipitation was carried out as described by Turck et al. (2004) and the immunoprecipitated DNA was concentrated using MinElute columns (Qiagen). Semi-quantitative polymerase chain reaction (SQ-PCR) amplifications $\left(56^{\circ} \mathrm{C}\right.$ annealing temperature with $45 \mathrm{~s}$ extension for 33 cycles) were performed using one-tenth of the volume from the immunoprecipitated DNA as template and Ampliqon Taq polymerase (Ampliqon, Roedovre, Denmark). PCR bands were quantified using a phosphoimager scanner (Molecular Dynamics) and Image Quant (Molecular Dynamics).

The following oligonucleotide sequences were used:

Region Afwd CGTACCCCCTCATGTTAGTAAAGATTTGC
rev GCTCTTTTAAAGCCACATAAACCGTAATACC
Region Bfwd GAAAGGCACACCATCTACTTAACTAATC
rev GTTTGGGATAATTACAGCTACACCCACTG
Region Cfwd ATGCTTAGAACTTCCTCGATTCATCTC
rev GAGATATTGACACGTGGGAAATCAACTC
Region Dfwd CCTCAGTGGGTGTAGCTGTAATTATCCC
rev CTTGTGTTAGATCTCGAAACCACTTAGGTC
Region Efwd CAATTAAGGGCTAATTGACTTTGATATACCG
rev CAACTTCTGCATCACAAAGAATAGCAAGC

Statistical analysis

In order to assess whether the variance between two sets of data was due to sampling bias or statistically relevant, a Student's $t$ test was performed with the wildtype, mutant and input data. The $t$ test compares the actual difference between two means in relation to the variation in the data. MS Excel (MicroSoft, USA) was used to compute the $t$ value. The significance threshold of the $t$ value commonly used is 0.05 . For values below 0.05 , there is a $95 \%$ probability of a significant difference of the two investigated data sets (Miller 1986). For example, values below 0.05 were obtained when ratios from the regions $\mathrm{C} / \mathrm{A}$ or $\mathrm{D} / \mathrm{A}$ from wild-type and input data were compared, showing that the wild-type enrichment was not simply due to sampling bias. Values above 0.05 were obtained for mutant and input data demonstrating that no region of the
DEFH125 promoter was specifically enriched under these conditions.

\section{Results and discussion}

Isolation of the DEFH125 promoter

Recent expression analysis of the Antirrhinum MADSbox protein DEFH125 revealed a pollen-specific expression. We isolated and sequenced a $2.5 \mathrm{kbp}$ fragment upstream of the putative DEFH125 ATG start codon in order to characterize its capacity to confer pollen-specific expression (DQ323884). We searched the promoter for the presence of CArG-box motifs, the conserved consensus sequence $\mathrm{CC}(\mathrm{A} / \mathrm{T})_{6} \mathrm{GG}$, known to mediate DNA binding of MADS-box proteins (Schwarz-Sommer et al. 1992). Three CArG-motifs were identified, allowing a change of one of the $\mathrm{CC}$ or $\mathrm{GG}$ nucleotides into an A/T (Fig. 1).

DEFH 125 promoter drives GUS expression in pollen and pollen tubes

To investigate the DEFH125 promoter activity, the $2.5 \mathrm{kbp}$ promoter fragment was fused to the Escherichia coli uidA gene, which encodes the $\beta$-glucuronidase (GUS) enzyme. Antirrhinum majus wild-type plants were transformed with Agrobacterium harboring the DEFH 125::GUS construct. Three independent transgenic plants (line 30, 47 and 102) were obtained. All lines revealed pollen-specific reporter gene expression and a wild-type-like phenotype. Line 30 and 102 showed GUS staining of $\sim 50 \%$ of the pollen, indicating that most likely a single transgene integrated into the genome. For further analysis, line 47 was selected, as here, all pollen were stained, probably due to at least two independent integration events. After selfing of the plants, progeny of line 47 was analyzed by staining 2 to 3 -week seedlings and flower buds at different stages. GUS color was detected in anthers of older flower buds that reached a length of $7 \mathrm{~mm}$ (Fig. 2a). No GUS staining was observed in sepals, petals and carpels before and after fertilization (Fig. 2a) and vegetative parts of the plant (data not shown). To precisely determine the temporal and spatial GUS expression conferred by the DEFH125 promoter, anthers from an early unicellular stage $(5 \mathrm{~mm}$ bud length) and an older, bicellular stage ( $7 \mathrm{~mm}$ bud length) were stained. After fixation and embedding, sections were observed under the microscope. GUS color was not detectable in uninucleate microspores (Fig. 2b). The onset of GUS staining appeared in an early bicellular stage when the first pollen mitosis was just accomplished and produced a vegetative and a generative cell (Fig. 2c, inset). As pollen development proceeded further, GUS color became stronger in the cytoplasm of the vegetative cell and was also detectable in pollen tubes that were germinated in vitro (Fig. 2d). 
CCCTTTATGGTATCAAAAATCTATACCAAACTCCCTCGTGGTATTTTTTATTTATTAGGATCCCCTTGTGTTTTCAGAAAAAAAAAAAAAACTATACACC - 2401 AАTTCTCCСATTCCСTTTATTATTACTCTCATATTTTCTATAAAGTTCTCCCAAATTTTCTCATACTATTAAAAACATGCACAAAACTCCCTCTTATAGT -2301 AAAAAAGATTTGCAA_CGTACCCCCTCATGTTAGTAAAGATTTGCATCAAACCCCCTCATATTAATAGAGTAAAATTGGTTATTCGTATTTAATAAAGGAT TTGACGCTAGATTTTGGAAACACGAGAGCATTTTGTGCAAAAAAAGTAATACGAGGAGTTTTGGTGCATAGTCTTTAAAACACGAGGGAATATTGTGCAA-2101 CAAAATAACACGAGAGAGTTTGTTGCAGGTCTTCTAAAACACAAATGGGTTTTATGCATTTTTCCCTAAATTTTATTTGATCCCAAGCTATTTTAATTTT -2001 ACTTATCTTATATTAAAATTAACAAAGAAGGGTTGAAAACTAAATTCAAGTTGTTAATGATAGAATTTTGCCGTTGTTAAATGCTAAAAGGTAATGCCGC - 1901 AGTAAGGAATTAGATCTACTTTGCATTGGGAAGTCTTATTAATTTTAGCATATGAGGTATTACGGTTTATGTGGCTTTAAAAGAGCAAATATATCTTAGA_-1801 rev A

CTGCAATTAAGAGTATTAATTTCAAAAAAACTCACCCAGACCACAGCCCGGGTGAGCCTTCAACGTGGCTCCGCCACTGCTTGAGAGCATGAAGGTCTTA-1701 AAACATAATATTGGTGATAGTCGCAGTTCTACCTCATATTTCAGAATTGCATTATCAATATGTTTTTAGGGTGTTTCAATATATGAATTTGAAAATAATG -1601 GAAATAGACAAGATTATTCATCACTAAATAAATTTTAATTGTGGGAAAATGTTTCTTTGTTTTTCATCAGAAAAAAATGCTTAATCTGAACCATATTCAA-1501 CArG3

CGGATGCCTGAAAAAGTAACATCTTCTTGTGAGAAAAAAGAAAAAATATAAAGAAAACTAAAAAAAGAAATATATATATAAAATTAAAAAAGGAAAGGAA-1401 ATATAAAAAAAGCTATCTGTGTACTATGTATGACGTTCAGCTTTAATTACTAGATAAAAACCCAAAAGGGAATGCATATCTATCTGTTTAGCTACAAGTT -1301 GTGTGACCTTTCCCAGATAATTTTTGATAAAACTTATAGGGATAATTACAGCTACACCTCCTAGGTTTGGTGTAATTACAGAGACCACCCCTGAAGTTTG - 1201 GGATATTACAGAGACCACCCCTGAGGTATGGCATAAACCTTACAACAAGGTCCATCTATTGAATTCATGGATAGAAACTTCCAATTTTACCCTTGGTCAA -1101 TTTCAATGAATATTATACAAAATTTGAATGGTTTCTTTATATAAAATAAATATCATTCTTTAAAGTCTACCAATACCTTTTTAAATACAAAAATAAGAAT - 1001 fWd B TATATTGTTGAAAAAAACTTAAAAGTCAAATTACATTTTTTTTTAAAAGAAAGGCACACCATCTACTTAACTAATCTATTTATTTAAATTTAATCGTAAA_901 TATTTAAAAACTCATAATATCATTTTTAATGTTTATAAATTATAGTTTGCCCATTAATATAAAAAATAAAAGAATACAATATGCTTAGAACTTCCTCGAT-801 $\longrightarrow$ TCATCTCAACAAAAAATGCACGAAAAATGAAACAAATTTCTTAACTTGCAAATTGCAATAATATTTTGGTACAAAATTTATTACAAATAAAAGAAAAAA - 70 AAAAAAAGGGAAAGCTCTTCAAACCAAAAAGAAAAATCATTATGTCTTTTGTAATTAACTTAATTTTTTAGATTCTCTAAAAAAAGTATGGAAGGGTATT - 601

ATTGTCTTTTATATGACATAAGTTAGTTACAGGAGAGGTCTCTGTAATTTCTCAAACTTCAGGGGAGGTCTCTGTAATTACCCCAAACCTCAGTGGGTGT-501
CArG2
AGd D

Fig. 1 Sequence and structure of the $D E F H 125$ promoter. The sequence of $2.5 \mathrm{kbp}$ of the $D E F H 125$ promoter is reported together with a fragment of the $D E F H 125$ coding region. Forward $(f w d)$ and reverse (rev) primers employed in the ChIP analysis are indicated. Letters $A-E$ correspond to the five amplified regions (see Fig. 2).

Upon self-pollination of line 47, pollen tubes growing through the style toward the ovules were also expressing GUS (data not shown). Taken together, our data show that DEFH125 promoter confers a pollen-specific GUS expression in Antirrhinum, starting after the first mitotic division of the microspore had occurred. Thus, the isolated $2.5 \mathrm{kbp}$ promoter fragment confers an expression identical to the endogenous DEFH125 protein expression pattern (Zachgo et al. 1997). Due to the small size of the generative cells, being embedded in the large vegetative cytoplasm, the GUS expression study did not allow to discriminate if the DEFH125 promoter also drives expression in the generative cells.

To check whether the $D E F H 125$ promoter activity is also conserved in distantly related species, we transformed the DEFH125::GUS construct into Arabidopsis thaliana. Fifteen transgenic T1 lines were obtained and analyzed; all of them showed a GUS signal confined exclusively to anthers at late floral stages (Fig. 2e).
Positions of three CArG-boxes are shown (CArG1, 2 and 3). They were identified allowing one substitution from the $\mathrm{CArG}$ consensus $\mathrm{CC}(\mathrm{A} / \mathrm{T})_{6} \mathrm{GG}$ in the $\mathrm{GG}$ or $\mathrm{CC}$ nucleotides. The putative $\mathrm{ATG}$ start codon is indicated by a black box

Analysis of anthers at different stages gave similar results as in Antirrhinum. No GUS color was detectable in microspores at the uninucleate microspore stage. However, at this stage, transgenic Arabidopsis plants showed a weak and transient GUS signal in the tapetum that was not detectable in Antirrhinum (compare Fig. 2b, f). As in Antirrhinum, onset of GUS staining was detectable from the early two cellular microspore stages onward (Fig. $2 \mathrm{~g}$ and inset) and was observed later in matured pollen and in in vitro germinated pollen tubes (Fig. 2h).

Analysis of the DEFH125 promoter revealed a conserved regulatory mechanism conferring pollen and pollen tube expression of DEFH125 in two species belonging to the asterid and rosid clades. Besides the conservation of the pollen-specific expression, minor regulatory differences seem to have evolved that lead to a short, transient tapetum activity of the DEFH125 promoter in Arabidopsis, not observed in Antirrhinum. The monocot MADS-box gene ZmMADS2, 

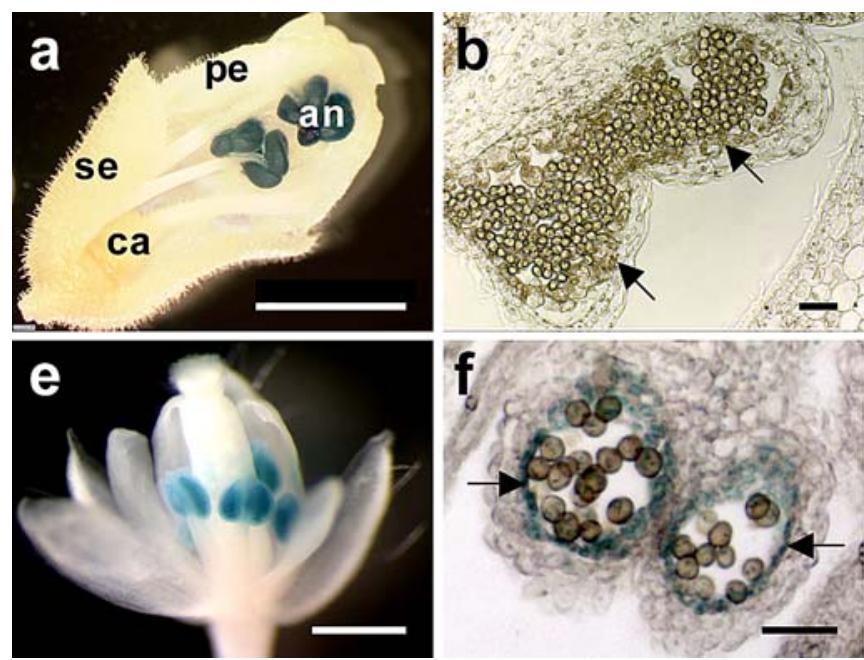

Fig. 2 DEFH125::GUS expression in Antirrhinum majus and Arabidopsis thaliana. Analysis of DEFH125::GUS expression in a-d Antirrhinum and e-h Arabidopsis. a An Antirrhinum flower with mature pollen (15 mm length) from the transgenic plant 47 shows only in anthers strong GUS staining. se sepal, pe petal, an anther, ca carpel. b Cross-section through a stained anther at the uninucleate stage (5-6 mm length) reveals the absence of GUS staining in uninucleate microspores and tapetum (indicated by arrows). $\mathbf{c}$ Cross-section through a stained pollen sac at a bicellular stage (10 mm length). Homogenous GUS staining is observed in all pollen. Inset shows a DAPI-stained pollen at the same stage to visualize the vegetative nucleus $(v n)$ and generative cell $(g c)$, indicated by white arrows. d Strong GUS staining of in vitro

a DEFH125 homolog from Zea mays, is also expressed in pollen and pollen tube but has evolved an additional function. ZmMADS2 expression is also transiently detectable in the endothecium and connective tissues of the anther (Schreiber et al. 2004). Functional analysis showed that this gene not only plays a role in pollen maturation but is also involved in anther dehiscence (Schreiber et al. 2004).

Determination of $D E F H 125$ promoter activity onset by DTA assay

The $D E F H 125$ promoter was used to drive expression of the bacterial $D T A$ gene in $A$. thaliana to conduct a highly sensitive cell ablation assay. The DTA protein is highly toxic to eukaryotic cells and a few molecules are sufficient to inhibit protein translation leading to cell death (Yamaizumi et al. 1978). In plants, tissue-specific promoters driving DTA gene expression have been successfully applied to determine the onset of the respective promoter activities (Day et al. 1995; Twell 1995). We analyzed 16 transgenic DEFH125::DTA T1 lines. All lines showed partial or complete male sterile phenotypes, which was caused by abortion of pollen. Otherwise, transgenic plants displayed a wild-type-like phenotype (data not shown). To determine from which stage on pollen development was affected, serial sections of anthers from different transgenic lines were prepared.
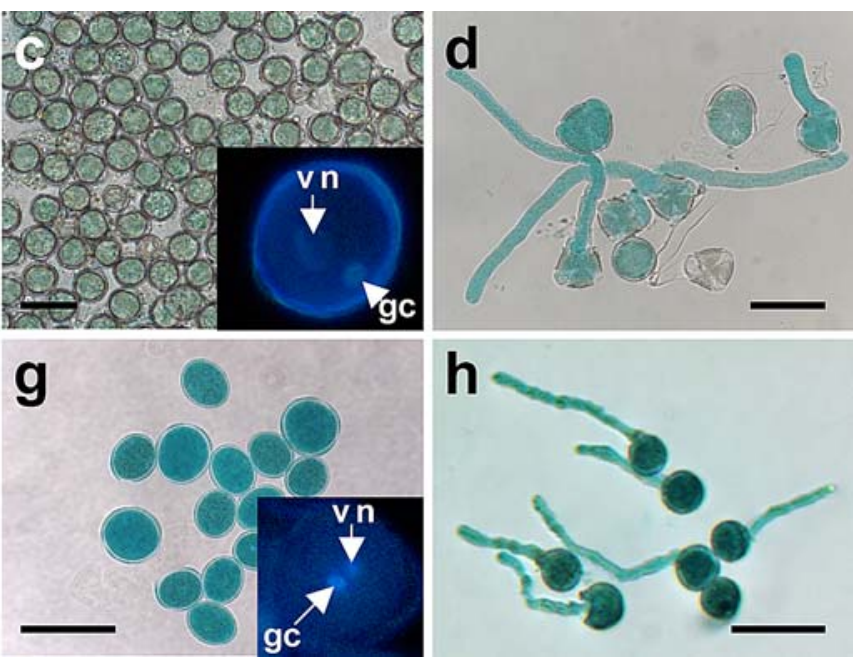

germinated pollen is visible in the cytoplasm of pollen and pollen tubes. e A mature flower bud (stage 12, according to Sanders et al. 1999) from a transgenic Arabidopsis line was stained, showing the GUS signal uniquely appearing in anthers. $\mathbf{f}$ Two pollen sacs from stained and sectioned anthers at the unicellular stage (stages 8-9). Whereas no staining was detectable in the microspores, a transient GUS expression was detectable in the tapetum (arrows). g A group of pollen at bicellular stage (stage 11) reveals a strong GUS signal. Inset shows a DAPI-stained pollen from the same stage with vegetative nucleus $(v n)$ and generative cell $(g c)$. $\mathbf{h}$ Transgenic, in vitro germinated pollen show strong GUS expression in cytoplasm and pollen tube. Bars $\mathbf{a}=5 \mathrm{~mm}, \mathbf{b}-\mathbf{d}=50 \mu \mathrm{m}, \mathbf{e}=500 \mu \mathrm{m}$, $\mathbf{f}-\mathbf{h}=25 \mu \mathrm{m}$

Compared to wild type, microspore development in the $D T A$ lines was only normal until the tetrad stage, after the meiotic divisions were completed (data not shown). The first detectable difference was found at the late uninucleate microspore stage. At this stage, wild-type microspores possess a big vacuole in the cytoplasm (Fig. 3a), which is broken down after the first mitosis (Yamamoto et al. 2003). However, this vacuole was not detectable in all microspores from the transgenic DTA lines (Fig. 3b). Microspores incapable of forming a vacuole never progressed further through development to conduct the first mitotic division that gives rise to the generative and vegetative cell. Instead, microspores lost their cytoplasm, collapsed and were visible as shrunken structures. Some DTA lines showed $\sim 50 \%$ of the pollen being aborted (Fig. 3c). These lines likely contained only one T-DNA copy, thus $50 \%$ of the haploid pollen carrying no transgene did develop normally in the pollen sacs. The DEFH125::GUS analysis showed a transient GUS expression in the Arabidopsis tapetum at the unicellular stage (Fig. 2f). Infered from this observation, it could be possible that DTA under the control of the DEFH 125 promoter is also expressed in the tapetum. However, degeneration of the tapetum in these transgenic plants occurred at a similar stage as in wild-type anthers and did not hinder development of $\sim 50 \%$ normal haploid pollen (data not shown). Other DTA lines, showing a complete ablation of all pollen, are presumed to contain more independent T-DNA copies (Fig. 3d). 


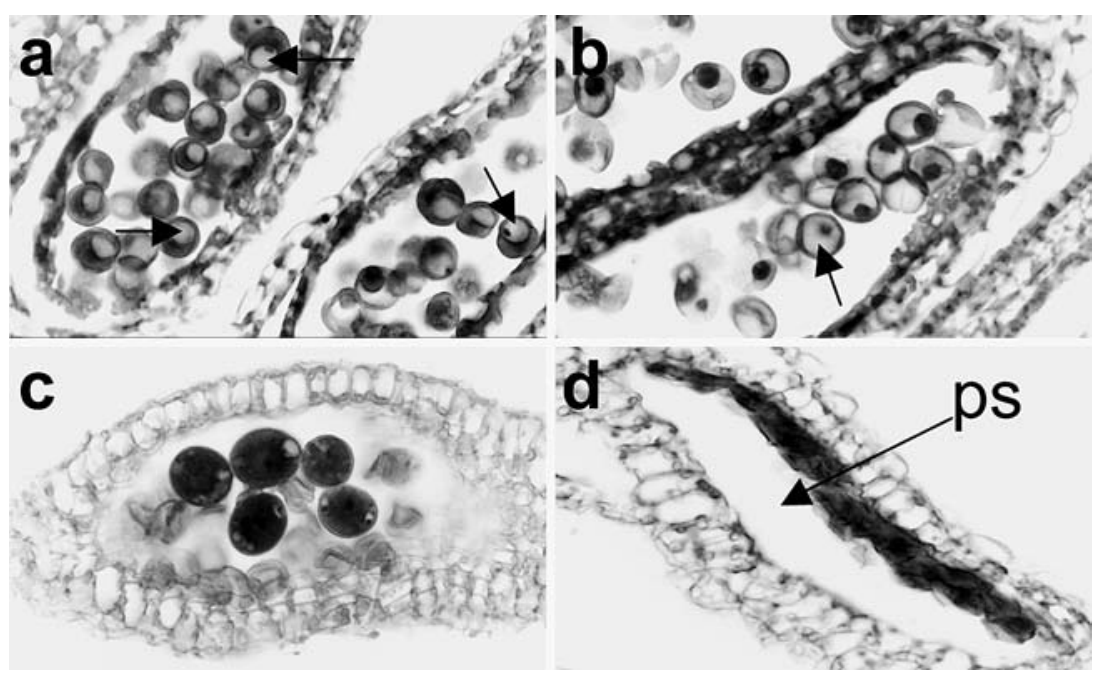

Fig. 3 Analysis of DEFH125::DTA in Arabidopsis plants. a Two pollen sacs from a sectioned wild-type Arabidopsis anther at a late uninucleate stage (stage 9), showing the presence of a large vacuole in microspores (arrows). b Pollen sacs from a DTA line at same stage as a. Most microspores in these pollen sacs are lacking an obvious vacuole (arrow). c Pollen sac from a DTA line at the

The DTA results demonstrate that microspores containing expressed $D T A$ are aborted before they progress into the bicellular stage. Due to the higher sensitivity of the DTA assay, onset of DEFH125 promoter activity could be defined to occur already at the unicellular stage, thus predating the onset of GUS expression detection observed at the bicellular stage. To summarize, the $D T A$ data support that DEFH125 promoter activity starts at the uninucleate stage in Arabidopsis, which may be similar in Antirrhinum. In pollen development, the first mitosis is an asymmetrical division and generates two daughter cells adopting different cell fates (Scott et al. 2004). As DEFH125 (i) expression starts before pollen mitosis I, the DEFH125 MADS-box gene might be involved in this decisive process. Other MADS-box genes from Arabidopsis, like AGL18 (Alvarez-Buylla et al. 2002) and $A G L 21$ (S. Xing and S. Zachgo, unpublished observation), are also expressed before and after pollen mitosis I, indicating the complexity and likely redundancy in the control of this process.

DEF and GLO proteins are expressed throughout petals and stamens but absent from microspores

Expression analysis of the Antirrhinum class B DEF and GLO proteins governing petal and stamen organogenesis revealed that both are expressed uniformly throughout the petal and stamen primordia during early floral stages (Zachgo et al. 1995, 2000; Fig. 4a, b). In this study, we analyzed the expression pattern during later stages, after the differentiation of floral organs has started. Figure $4 \mathrm{c}$, d shows immunolabeled sections through young flower buds with anthers containing bicellular stage (stage 11), showing about $50 \%$ dead pollen and $50 \%$ normally developed pollen. d Pollen sac from another DTA line at a comparable stage as in $\mathbf{c}$; however, likely due to a high copy number insertion, pollen have not at all developed. Dead, aborted pollen are lining up along the anther wall. Tissue was stained with Naphthol Blue Black. Bars $25 \mu \mathrm{m}$

young microsporocytes that will soon undergo meiosis, form tetrads and release haploid microspores. DEF and GLO proteins are still expressed throughout the petals. However, in young stamens, expression is restricted to the filaments, connective tissue and vasculature, and no signal could be detected in the microsporocytes (Fig. 4c, d). Although DEF/GLO are required for stamen organogenesis, a lack of expression in microsporocytes indicates that the formation of a vegetative and generative cell is likely under the control of other regulatory genes. Figure 4e, f shows an advanced floral stage, after accomplishment of meiosis and the first mitotic division, in which bicellular microspores were generated that are visible as almost mature pollen in the pollen sacs. DEF and GLO protein expression is still absent from the pollen and only detectable in the filament, connective tissue and vasculature. In older petals and filaments, expression is strongest in epidermal cell layers. Analysis of the $D E F H 125$ and $D E F / G L O$ expression at older developmental stages thus revealed a complementary expression pattern in stamens. This observation raised the question, whether the DEF/GLO heterodimer could be involved in regulation of DEFH125 by binding its promoter and acting as a repressor of the DEFH125 transcription.

Establishment of the ChIP technique to analyze an in vivo interaction of the $\mathrm{DEF} / \mathrm{GLO}$ heterodimer at the DEFH125 promoter

The ChIP technique delivers the proof of in vivo protein/DNA interactions. The technique has been initially applied to study transcription factor/DNA interactions 
Fig. 4 DEF and GLO protein expression in older floral stages. DEF protein (a, c, d) and GLO protein expression patterns (b, d, f) in wild-type Antirrhinum buds were detected on longitudinal sections using antiDEF and anti-GLO sera. a, b

Young stages with undifferentiated floral primordia. c, d Organ differentiation has started and expression is detectable in different petal and stamen tissues but no expression could be observed in the microsporocytes developing in the microsporangia. $\mathbf{e}, \mathbf{f}$ Similarly, older flowers show no $\mathrm{DEF} / \mathrm{GLO}$ expression in almost mature pollen. se sepal, pe petal, st stamen, $c a$ carpel, $p s$ pollen sac, $f i$ filament. Bars $200 \mu \mathrm{m}$.

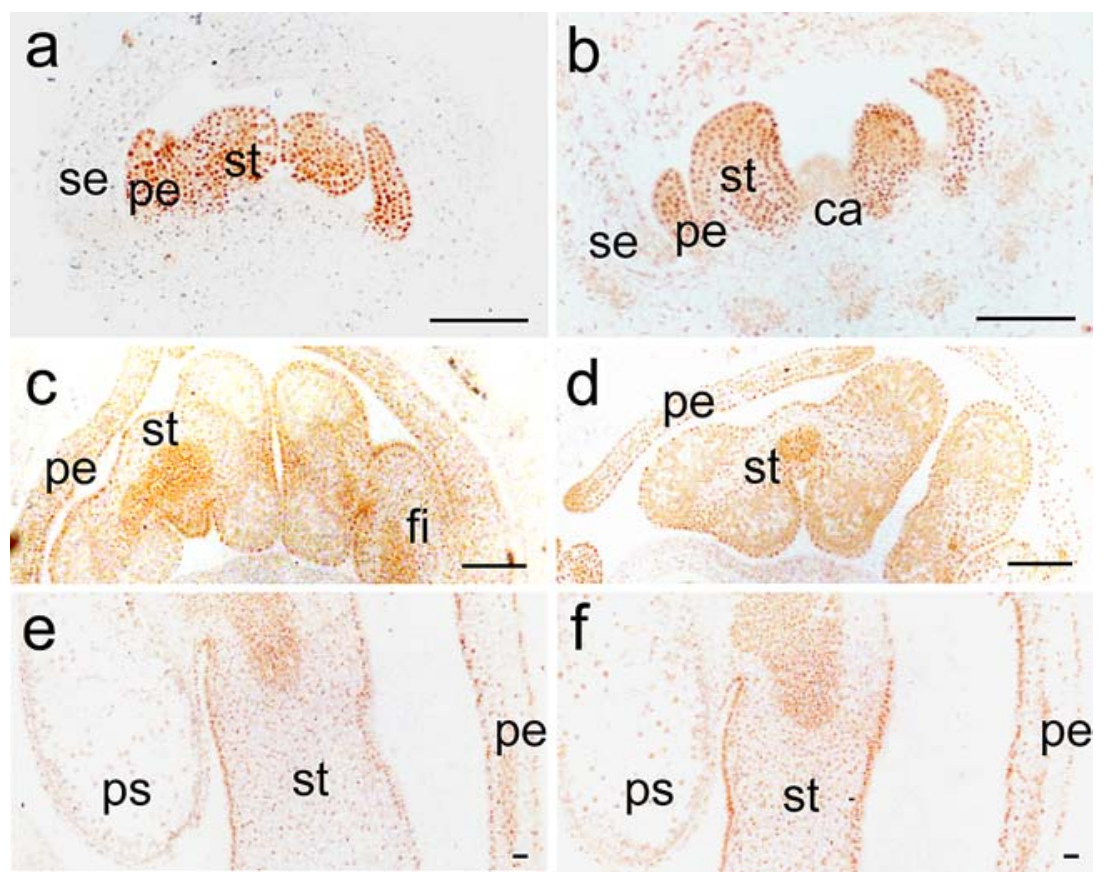

in Drosophila (Orlando et al. 1997). Recently, the technique has also been employed for plant protein/ DNA studies (Turck et al. 2004; William et al. 2004; Gomez-Mena et al. 2005). We were intrigued by the observation that the DEF protein binds to a DEFH125 promoter region of about $1 \mathrm{kbp}$ (Müller 2003). Therefore, we investigated the binding of both, the DEF and GLO proteins, to analyze precisely their in vivo interaction with a $2.3 \mathrm{kbp}$ long $D E F H 125$ promoter region by conducting an in-depth ChIP analysis. After fixation of Antirrhinum plant material, nuclei were extracted and DNA fragments of an average size of 300-400 bp were generated by random shearing. Immunoprecipitation was carried out with anti-DEF and anti-GLO sera that were successfully used in immunolocalization studies (Zachgo et al. 1995, 2000). The enrichment of specifically bound DNA sequences in the immunoprecipitated DNA pool was determined by SQ-PCRs, for which an example is given in Fig. 5b. To prove the reliability of the data and to allow a comparison be- tween the different analyzed promoter regions, several control steps were included. To circumvent the intrinsic variability of the PCR, each ChIP experiment and consecutive PCRs were conducted at least six times. To discriminate between real in vivo protein/DNA interactions and amplification of non-specific background, and thus to demonstrate serum specificity, each experiment was conducted in parallel with the material from the $D E F$ and $G L O$ null-mutants, def-gli and $g l o$, respectively. For determination of DEF/GLO DNA-binding sites in the DEFH125 promoter, four partially overlapping promoter regions (B-E) were considered (Fig. 5a). Each fragment spans about $400 \mathrm{bp}$ and together, they cover about $1,000 \mathrm{bp}$ upstream of the putative start codon. Region A, located $\sim 2.3 \mathrm{kbp}$ upstream of the ATG, was shown to be suitable as a background control. Within this interval, the overall chromatin structure is supposed to be still comparable and thus its impact on the shearing and immunoprecipitation procedure similar (Schwartz et al. 2005).
Fig. 5 Overview on the DEFH125 ChIP experiments. a Investigated regions $\mathrm{A}-\mathrm{E}$ in the DEFH 125 promoter, as well as position and sequence of the CArG1 and CArG2 motifs, are indicated. b Representative example of a SQ-PCR conducted on 'wild-type', 'mutant' and 'input' material. Immunoprecipitation was carried out with anti-DEF serum. Primers for amplification of regions $\mathrm{A}-\mathrm{E}$ are reported in Fig. 1

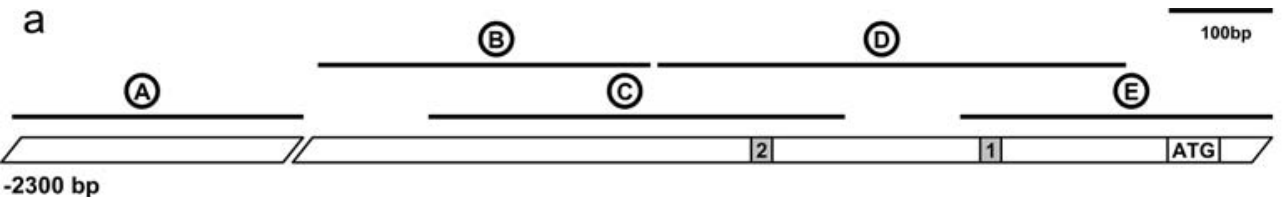

ATG transcription start codon 2 CArG2: ACTTAAATGG 1 CArG1: ACAATTAAGG

b

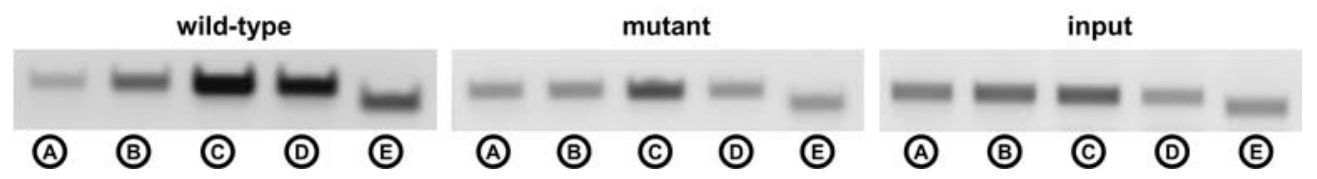



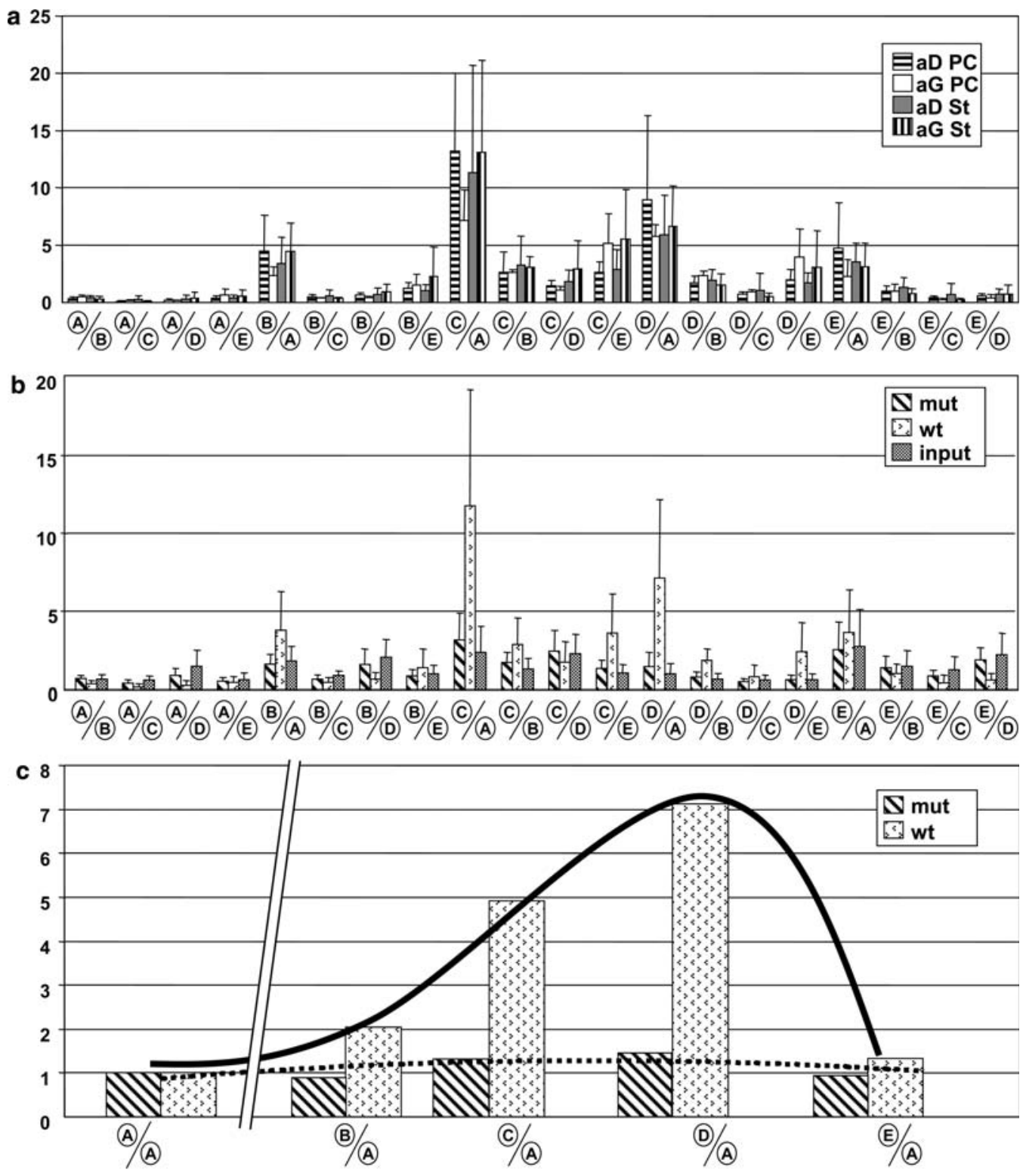

(B)

(D)

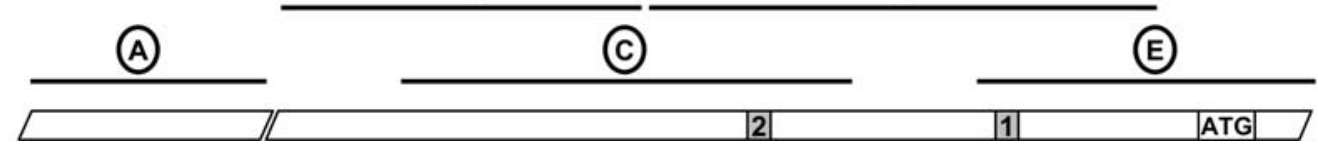

Fig. 6 Binding of DEF/GLO to the $D E F H 125$ promoter. a The chart illustrates the average values of all band intensity ratios of the ChIP experiments conducted on petals and stamens using antiDEF (aD Pe, aD St) and anti-GLO serum (aG Pe, aG St), respectively. b 'Wild-type' ( $w t)$ columns correspond to average values of four sets of data: stamen and petal material treated with the two anti-sera, reported separately in Fig. 5a. Values corresponding to 'mutant' (mut) comprise results of experiments conducted with anti-DEF serum and anti-GLO serum on def-gli

For normalization of primer strength, control SQPCRs were conducted in parallel with 'input' DNA (Fig. 5b). Input DNA originated from an aliquot taken after the sonication step, but before the immunoprecipitation, and was diluted to a concentration compa- and glo mutant inflorescences, respectively. As no significant differences were noted between these two sets of data they were reported together (16 replicates in total). Input (input) values are the average of 21 independent SQ-PCRs. c Further on, only the set of values generated in relation to region $\mathrm{A}$ were considered and divided by the input values for normalization. A bell-shaped curve (top enrichment corresponding to region D) for experiments using wild-type material could be extrapolated. An almost flat line reflects the lack of enrichment in the mutant experiments

rable to the immunoprecipitated DNA. To determine the relative fragment enrichment, PCR band intensities were quantified and ratio values were pairwise calculated within each experiment. Mean values of ratios and the corresponding standard deviations were calculated. 
Furthermore, a statistical approach, the Student's $t$ test (see Materials and methods), was conducted to assess whether differences between data sets from wild-type, mutant and input were due to a specific enrichment or caused by sampling bias.

DEF and GLO bind in vivo to the DEFH125 promoter

The ChIP experiments were conducted in parallel with petal and stamen material harvested from $\sim 1 \mathrm{~cm}$ long wild-type flowers and with inflorescences from $D E F$ and $G L O$ mutants using anti-DEF and anti-GLO sera. After quantification of the PCR repetitions, ratios between all five investigated regions in one experiment were calculated and are shown in Fig. 6a. The clear tendency of region $\mathrm{D}$ to be enriched with respect to the other regions was confirmed by further processing of the data (see also PCR gel pictures in Fig. 5b). Very similar ratio values were obtained in petals and stamens using both antiDEF and anti-GLO sera, respectively (Fig. 6a). Therefore, these four data sets were treated together as one wild-type group for further normalization steps. Similarly, data collected from mutant tissues showed negligible variations (data not shown) and were thus treated as one set of data (Fig. 6b). Applying a Student's $t$ test, the specificity of the observed enrichment of regions $\mathrm{C}$ and $\mathrm{D}$ in wild-type tissues was verified. On the contrary, calculated ratios from input and mutant data were low (Fig. 6b). Furthermore, region A showed the lowest enrichment in the wild-type experiments, demonstrating its applicability as a background control. Together, our data provide evidence that DEF and GLO proteins bind in vivo to the same area in the DEFH125 promoter. It is therefore likely that they bind the DNA as a heterodimer, as has been shown by former in vitro binding studies (Tröbner et al. 1992; Zachgo et al. 1995).

To correct for the differences in primer strength, values depicted in Fig. $6 \mathrm{~b}$ from wild type and mutant were divided by the corresponding input value. For better visualization of the normalized data, Fig. 6c shows the set of values determined in relation to the background control (region A). The curve extrapolated from the wild-type data approximates a Gaussian distribution, describing the relation between enrichment of a region and its distance from the binding site (Fig. 6c). For the mutant data set, an almost flat line could be projected. No region of the DEFH125 promoter can be specifically immunoprecipitated in the absence of the investigated protein in the respective mutants, thus demonstrating the specificity of the serum. The peak of the curve is represented by region $\mathrm{D}$ and a slightly reduced enrichment was detected for region C. Enrichment values decrease for regions $\mathrm{B}$ and $\mathrm{E}$ and reach the lowest value for region $\mathrm{A}$. The majority of the sheared fragments spans about $400 \mathrm{bp}$. However, as random sonication also generates lower amounts of longer fragments, these can still be precipitated and PCR amplified with primers not spanning the binding site and thus explain the slight enrichment observed for regions B and $\mathrm{E}$.

Mapping of the DEF/GLO binding sites defines CArG2 as a likely interaction site

Given that the PCR primers were designed to amplify partially overlapping $400 \mathrm{bp}$ long regions, the ChIP data allowed defining the DEF/GLO binding region more precisely. Regions $\mathrm{C}$ and $\mathrm{D}$ displayed the strongest enrichment, whereas values for region B dropped down to a level more similar to that observed for region $\mathrm{A}$, the background control. This allows restricting the binding area for the DEF/GLO heterodimer to an area of $\sim 200 \mathrm{bp}$ in the DEFH125 promoter, located $220 \mathrm{bp}$ upstream of the putative start codon. This area contains the CArG2 motif (ACTTAAATGG) showing one single mismatch from the conserved $\mathrm{CArG}$ consensus motif $\mathrm{CC}\left(\mathrm{A} / \mathrm{T}_{6}\right) \mathrm{GG}$. Besides recognizing the CArG1 motif in the $D E F$ promoter that fully matches the CArG-box consensus sequence (CCTTTTTAGG), slight deviations from the consensus were also shown to be recognized by DEF/GLO and AP3/PI heterodimers (Tröbner et al. 1992; Tilly et al. 1998; Hill et al. 1998). The ChIP data demonstrate specificity of the interaction in region $\mathrm{D}$ comprising CArG2, as other tested CArG-boxes were not promiscuously bound by the DEF/GLO heterodimer. One additional motif (CArG3, see Fig. 1) was identified upstream of CArG2 showing similar deviations from the conserved CArG-box consensus. However, in spite of sequence similarity, no specific enrichment could be observed in ChIP experiments (data not shown). This observation further strengthens the interaction specificity that might be conferred by additional cofactors and/or a specific chromatin structure to bind to the region containing $\mathrm{CArG} 2$.

\section{Conclusion}

In this study, we characterized the DEFH125 promoter by different reporter gene studies and demonstrate its usability for pollen-specific studies in Antirrhinum and Arabidopsis. Furthermore, being intrigued by the complementary $D E F H 125$ and $D E F / G L O$ expression pattern in stamens, we conducted a thorough ChIP binding analysis showing that the DEFH125 promoter is bound in vivo by $\mathrm{DEF} / \mathrm{GLO}$. We demonstrate that the ChIP technique can be exploited to map the DEF/ GLO binding site down to a region of about $200 \mathrm{bp}$ in the DEFH125 promoter. The whole investigated promoter fragment comprises several CArG-boxes, short DNA-binding motifs for MADS-box proteins. However, only one motif within the $200 \mathrm{bp}$ region, CArG2, seems to be directly recognized by the heterodimer, whereas other similar CArG-box motifs did not contribute to a specific in vivo interaction. So far, maintenance and up-regulation of later class B and also class 
C MADS-box gene expression by an autoregulatory loop has been interfered from genetic and biochemical studies conducted in Antirrhinum and Arabidopsis (Zachgo et al. 1995; Jack et al. 1994; Tilly et al. 1998; Gomez-Mena et al. 2005). Our in vivo ChIP experiments and expression studies suggest that the class B MADS-box proteins DEF and GLO could also be involved in the direct repression of the expression of another MADS-box gene, namely DEFH125, and could thereby contribute to the formation of mature male gametophytes.

Acknowledgements The authors thank Dr. Hans Sommer, MPIZ, for support with the molecular work. A.L. received a scholarship from the DFG (Graduierten Kolleg, 'Molecular analysis of developmental processes'). This work was supported by a grant from the DFG to S.Z. (ZA 259/3-1).

\section{References}

Alvarez-Buylla ER, Liljegren SJ, Pelaz S, Gold SE, Burgeff C, Ditta GS, Vergara-Silva F, Yanofsky MF (2002) MADS-box gene evolution beyond flowers: expression in pollen, endosperm, guard cells, roots and trichomes. Plant J 24:457-466

Bate N, Twell D (1998) Functional architecture of a late pollen promoter: pollen-specific transcription is developmentally regulated by multiple stage-specific and co-dependent activator elements. Plant Mol Biol 37:859-869

Becker D, Kemper E, Schell J, Masterson R (1992) New plant binary vectors with selectable markers located proximal to the left T-DNA border. Plant Mol Biol 20:1195-1197

Bey M, Stüber K, Fellenberg K, Schwarz-Sommer Z, Sommer H, Saedler H, Zachgo S (2004) Characterization of Antirrhinum petal development and identification of target genes of the class B MADS box gene DEFICIENS. Plant Cell 16:3197-3215

Chen Y-CS, McCormick S (1996) Sidecar pollen, an Arabidopsis thaliana male gametophytic mutant with aberrant cell divisions during pollen development. Development 122:3243-3253

Clough SJ, Bent AF (1998) Floral dip: a simplified method for Agrobacterium-mediated transformation of Arabidopsis thaliana. Plant J 16:735-743

Coen ES, Meyerowitz EM (1991) The war of the whorls: genetic interactions controlling flower development. Nature 353:31-37

Day CD, Galgoci BF, Irish VF (1995) Genetic ablation of petal and stamen primordia to elucidate cell interactions during floral development. Development 121:2887-2895

Egea-Cortines M, Saedler H, Sommer H (1999) Ternary complex formation between the MADS-box proteins SQUAMOSA, DEFICIENS and GLOBOSA is involved in the control of floral architecture in Antirrhinum majus. EMBO J 18:5370-5379

Gomez-Mena C, de Folter S, Costa MM, Angenent GC, Sablowski $\mathrm{R}$ (2005) Transcriptional program controlled by the floral homeotic gene AGAMOUS during early organogenesis. Development 132:429-438

Heidmann I, Efremova N, Saedler H, Schwarz-Sommer Z (1998) A protocol for transformation and regeneration of Antirrhinum majus. Plant J 13:723-728

Hill TA, Day CD, Zondlo SC, Thackeray AG, Irish VF (1998) Discrete spatial and temporal cis-acting elements regulate transcription of the Arabidopsis floral homeotic gene APETALA3. Development 125:1711-1721

Honma T, Goto K (2001) Complexes of MADS-box proteins are sufficient to convert leaves into floral organs. Nature 409:525-529

Honys D, Twell D (2003) Comparative analysis of the Arabidopsis pollen transcriptome. Plant Physiol 132:640-652

Honys D, Twell D (2004) Transcriptome analysis of haploid male gametophyte development in Arabidopsis. Genome Biol 5:R85
Jack T, Fox GL, Meyerowitz EM (1994) Arabidopsis homeotic gene $A P E T A L A 3$ ectopic expression: transcriptional and posttranscriptional regulation determine floral organ identity. Cell 76:703-716

McCormick S (2004) Control of male gametophyte development. Plant Cell 16(Suppl):S142-S153

Miller R (1986) Beyond ANOVA, basics of applied statistics. Wiley, New York

Müller BM (2003) Doktorarbeit (PhD thesis), University of Cologne

Orlando V, Strutt H, Paro R (1997) Analysis of chromatin structure by in vivo formaldehyde cross-linking. Methods 11:205214

Pina C, Pinto F, Feijó JA, Becker JD (2005) Gene family analysis of the Arabidopsis pollen transcriptome reveals biological implications for cell growth, division control, and gene expression regulation. Plant Physiol 138:744-756

Sanders PM, Bui AQ, Weterings K, McIntire KN, Hsu Y-C, Lee PY, Truong MT, Beals TP, Goldberg RB (1999) Anther developmental defects in Arabidopsis thaliana male-sterile mutants. Sex Plant Reprod 11:297-322

Schiefthaler U, Balasubramanian S, Sieber P, Chevalier D, Wisman E, Schneitz K (1999) Molecular analysis of NOZZLE, a gene involved in pattern formation and early sporogenesis during sex organ development in Arabidopsis thaliana. Proc Natl Acad Sci USA 96:11664-11669

Schreiber DN, Bantin J, Dresselhaus T (2004) The MADS box transcription factor ZmMADS2 is required for anther and pollen maturation in maize and accumulates in apoptotic bodies during anther dehiscence. Plant Physiol 134:1069-1079

Schwartz YB, Kahn TG, Pirrotta V (2005) Characteristic low density and shear sensitivity of cross-linked chromatin containing polycomb complexes. Mol Cell Biol 25:432-439

Schwarz-Sommer Z, Huijser P, Nacken W, Saedler H, Sommer H (1990) Genetic control of flower development by homeotic genes in Antirrhinum majus. Science 250:931-939

Schwarz-Sommer Z, Hue I, Huijser P, Flor P, Hansen R, Tetens F, Lönnig WE, Saedler H, Sommer H (1992) Characterization of the Antirrhinum floral homeotic MADS-box gene DEFICIENS: evidence for DNA binding and autoregulation of its persistent expression throughout flower development. EMBO J 11:251263

Scott RJ, Spielman M, Dickinson HG (2004) Stamen structure and function. Plant Cell 16(Suppl):S46-S60

Sommer H, Beltrán JP, Huijser P, Pape H, Lönnig WE, Saedler H, Schwarz-Sommer Z (1990) DEFICIENS, a homeotic gene involved in the control of flower morphogenesis in Antirrhinum majus: the protein shows homology to transcription factors. EMBO J 9:605-613

Sorensen AM, Krober S, Unte US, Huijser P, Dekker K, Saedler H (2003) The Arabidopsis ABORTED MICROSPORES (AMS) gene encodes a MYC class transcription factor. Plant J 33:413423

Theißen G, Saedler H (2001) Floral quartets. Nature 409:469-471

Tilly JJ, Allen DW, Jack T (1998) The CArG boxes in the promoter of the Arabidopsis floral organ identity gene APET$A L A 3$ mediate diverse regulatory effects. Development 125:1647-1657

Tröbner W, Ramirez L, Motte P, Hue I, Huijser P, Lönnig WE, Saedler H, Sommer H, Schwarz-Sommer Z (1992) GLOBOSA: a homeotic gene which interacts with DEFICIENS in the control of Antirrhinum floral organogenesis. EMBO J 11:4693-4704

Turck F, Zhou A, Somssich IE (2004) Stimulus-dependent, promoter-specific binding of transcription factor WRKY1 to its native promoter and the defense-related gene PcPRI-1 in parsley. Plant Cell 16:2573-2585

Twell D (1995) Diphtheria toxin-mediated cell ablation in developing pollen: vegetative cell ablation blocks generative cell migration. Protoplasma 187:144-154

William DA, Su Y, Smith MR, Lu M, Baldwin DA, Wagner D (2004) Genomic identification of direct target genes of LEAFY. Proc Natl Acad Sci USA 101:1775-1780 
Wilson ZA, Morroll SM, Dawson J, Swarup R, Tighe PJ (2001) The Arabidopsis MALE STERILITY1 (MS1) gene is a transcriptional regulator of male gametogenesis, with homology to the PHD-finger family of transcription factors. Plant J 28:27-39

Yamaizumi M, Mekada E, Uchida T, Okada Y (1978) One molecule of diphtheria toxin fragment A introduced into a cell can kill the cell. Cell 15:245-250

Yamamoto Y, Nishimura M, Hara-Nishimura I, Noguchi T (2003) Behavior of vacuoles during microspore and pollen development in Arabidopsis thaliana. Plant Cell Physiol 44:1192-1201

Yang WC, Ye D, Xu J, Sundaresan V (1999) The SPOROCYTELESS gene of Arabidopsis is required for initiation of sporogenesis and encodes a novel nuclear protein. Genes Dev $13: 2108-2117$
Zachgo S, de Andrade Silva E, Motte P, Tröbner W, Saedler H, Schwarz-Sommer Z (1995) Functional analysis of the Antirrhinum floral homeotic DEFICIENS gene in vivo and in vitro by using a temperature-sensitive mutant. Development 121:2861-2875

Zachgo S, Saedler H, Schwarz-Sommer Z (1997) Pollen-specific expression of $D E F H 125$, a MADS-box transcription factor in Antirrhinum with unusual features. Plant J 11:1043-1050

Zachgo S, Perbal MC, Saedler H, Schwarz-Sommer Z (2000) In situ analysis of RNA and protein expression in whole mounts facilitates detection of floral gene expression dynamics. Plant $\mathbf{J}$ 23:697-702 